\title{
Microfinance en Chine et en Inde : une « discipline » diversement appropriée
}

\section{Cyril Fouillet et Thierry Pairault}

\section{(2) OpenEdition \\ 1 Journals}

Édition électronique

URL : http://journals.openedition.org/ei/233

DOI : 10.4000/ei.233

ISSN : 2553-1891

Éditeur

Association Économie et Institutions

Édition imprimée

Date de publication : 30 mai 2010

Pagination : 117-140

ISSN : 1775-2329

\section{Référence électronique}

Cyril Fouillet et Thierry Pairault, « Microfinance en Chine et en Inde : une « discipline » diversement appropriée », Économie et institutions [En ligne], 14 | 2010, mis en ligne le 31 janvier 2013, consulté le 19 avril 2019. URL : http://journals.openedition.org/ei/233 ; DOI : 10.4000/ei.233 


\title{
Cyril Fouillet $^{1}$ et Thierry Pairault ${ }^{2}$
}

\author{
Microfinance en Chine et en Inde : une " discipline " \\ diversement appropriée ${ }^{3}$
}

\section{Introduction}

En octobre 2006, le professeur Muhammad Yunus et l'institution de microfinance qu'il a fondée, la Grameen Bank, recevaient le prix Nobel de la paix pour "leurs efforts pour créer un développement économique et social par le bas " et parce qu'ils " ont montré que les pauvres peuvent travailler à leur propre développement et que le micro-crédit a démontré qu'il est une importante force de libération des sociétés où les femmes en particulier doivent se battre contre des conditions économiques et sociales répressives " (The Norwegian Nobel Institute, 2006). La microfinance est pourtant loin d'être cette panacée tant vantée par les médias et les organisations internationales (Fernando, 2006 ; Servet, 2006). Perpétuellement réinterprétée et réappropriée, la microfinance peut échapper au contrôle de ses promoteurs ainsi qu'aux objectifs économiques et sociaux qui lui sont souvent assignés. Ainsi, la sémantique des bailleurs de fonds, des chercheurs en sciences sociales et des praticiens travaillant pour ou sur la microfinance révèle le plus souvent des visées normatives (la finance informelle est mauvaise, etc.) et une perspective évolutionniste (toutes les pratiques financières doivent à terme se fondre dans le Système). Encourager des projets de microfinance manifeste-t-il que

\footnotetext{
1 Université d'Oxford, School of Interdisciplinary Area Studies, 12, Bevington Road, OX2 L6H Oxford, Angleterre. Cyril Fouillet est également associé avec le Centre Européen de Recherche en Microfinance (CERMi-ULB) et membre du projet ANR les Sud Rural Employment and Microfinance (RUME-IEDES). cyril.fouillet@area.ox.ac.uk

2 CNRS/EHESS, CECMC, 54, bd Raspail, 75006 Paris, France. pairault@ehess.fr.

3 Nous tenons à remercier les trois évaluateurs anonymes dont la lecture attentive et les suggestions très constructives ont permis une amélioration substantielle du texte. Nous remercions également la Fondation Philippe Wiener-Maurice Anspach et l'Institut Français de Pondichéry (UMIFRE 21 CNRS-MAEE) dont le soutien financier a été déterminant pour la partie relative à l'Inde.
} 
les États adhèrent sans sourciller à ces préconceptions, que les acteurs locaux puissent à leur tour y rallier ou, inversement, s'en détourner au profit d'autres ambitions?

En s'appuyant sur les exemples chinois et indiens - deux grands marchés potentiels pour les professionnels de la microfinance - , nous nous demanderons dans cet article comment, au-delà d'une certaine normalisation, la microfinance donne lieu à des modes d'appropriation très distincts. Nous nous interrogerons sur le rôle de l'État central comme "passeur " de ce mot ainsi que sur la capacité et la liberté d'adaptation des organisations et des autorités locales qui les mettent en œuvre. Pour en rendre compte, nous reviendrons dans une première partie sur la vision normative que draine l'expression " microfinance ". Puis, dans une deuxième et troisième parties, nous décrirons la circulation de ce nouveau vocable en Chine et en Inde. Notre conclusion souligne qu'au-delà d'une certaine normalisation des techniques financières, la microfinance a également à s'accommoder d'une pluralité de pratiques tant politiques, que sociales voire religieuses.

\section{Une vision normative assumée}

La microfinance ne se présentait pas comme thématique à part entière avant 1997, année du lancement de la campagne du Sommet mondial du microcrédit ${ }^{4}$; auparavant seuls apparaissaient des travaux sur le thème du crédit rural, des tontines et du financement des petites exploitations agricoles ou de microentreprises. Cette évolution est intéressante car elle correspond à un certain déclin d'un autre concept, celui de coopérative. Comme le fait remarquer Panu Kalmi (2007, p. 638-642) dans une étude sur le concept de coopérative dans la littérature économique, cette relative disparition est peut être à rapprocher de l'intérêt nouveau des chercheurs pour des solutions plus ancrées dans l'économie locale comme c'est le cas en microfinance. Néanmoins selon lui, ces glissements se font plutôt l'écho d'un changement de paradigme économique : on serait passé d'une économie orientée principalement

4 La campagne du Sommet du microcrédit a été lancée en 1997 à Washington avec comme objectif pour l'année 2015 de " s'assurer que 175 millions des familles les plus pauvres au monde, particulièrement les femmes membres de ces familles, reçoivent du crédit leur permettant d'exercer une activité économique indépendante, ainsi que d'autres services financiers et commerciaux. "(Daley-Harris, 2007, p. 3). Les personnalités impliquées dans les différentes chaires de cette campagne proviennent de tous les horizons : firmes multinationales (Monsanto), groupes bancaires (Citigroup), personnes politiques (Hillary Clinton), organisations de la société civile (FINCA International, Women's World Banking), fondations (Fondations des Nations Unies, Charles Stewart Mott, George Soros), etc. 
par des analyses institutionnelles à une économie néoclassique qui minimise l'importance des éléments institutionnels et donne plus de crédit aux solutions optimales. La microfinance participe pleinement à ce processus et les pratiques microfinancières s'accompagnent le plus souvent de règles très strictes, d'un protocole minutieux imposant une véritable doctrine de la discipline financière. Faisant suite aux premières tentatives du Programme des Nations Unies pour le développement (PNUD) dans les provinces chinoises du Henan et du Hebei, un rapport concluait que la : "La microfinance représente un type d'intervention distincte d'autres activités par le besoin de discipline et de rendre compte de l'assurance à créer des services financiers soutenables. L'expérience mondiale est concluante sur le fait que si ce service est combiné avec d'autres efforts complexes (systèmes d'approvisionnement d'eau, santé, éducation, $\mathrm{TVE}^{5}$ développement, irrigation, etc.), les clients seront aptes à considérer le composant de prêt plus comme un programme gouvernemental de bien-être que comme un effort de discipline à établir des services soutenables " (UNPD, 1997, p. 11-12).

Posant la question des conditions du crédit, le Consultative Group to Assist the Poorest (CGAP) répondait ainsi en 2002 : "Le succès requiert à la fois la discipline du client (rembousement régulier) et des institutions (pratiques soutenables)" (World Bank, 2002 , p. 1). Dans un autre texte dont l'objectif est d'examiner "Le microcredit est un élement parmi d'autres d'un menu d'interventions possibles pour générer de l'emploi et des revenus ainsi que pour alléger la pauvreté, y compris la pauvreté temporaire des situations d'après crise et le noyau dur de la pauvreté à long terme " (CGAP, 2002 , p. 1), le mot discipline revient douze fois dans un texte de quatorze pages dont neuf fois dans la seule quatrième page traitant des éléments indispensables à la réussite d'un programme de microcrédit. Si l'on regarde de près, la discipline du client apparaît être la clef de voute des IMF.

"Le microcrédit réussi repose sur deux principes de base: la discipline du client et celle des i nstitutions. (...) La première signifie que les pauvres prennent leurs responsabilités en acceptant et en réalisant le remboursement à temps du principal et de la somme des intérêts qui couvrent le plein coût du service " (p. 3).

Le message est clair ; la discipline de la clientèle est d'autant plus indispensable qu'elle conditionne la discipline des institutions et leur pérennité.

5 TVE = Towns and villages entreprises. C'est la traduction du chinois xiangzhen qiye traduite en français par "entreprises de bourgs et de villages". 
Avec la microfinance, c'est un autre monde que l'on tente de construire, un monde où le néocolonialisme semble laisser place au néolibéralisme. Un monde où la Banque mondiale via le CGAP et une pléiade d'organisations de la société civile, d’institutions financières, etc. appliquant consciemment ou non des structures qui dessinent une réalité allant dans le sens d'une mondialisation financière néolibérale. Une citation de Mohammad Yunus est très claire à ce sujet : "Le crédit sans une stricte discipline n'est rien d'autre que de la charité. Celle-ci n'aide pas à sortir de la pauvreté. La pauvreté est une maladie qui à un effet paralysant, mental et corporel. Un programme sensé d'allégement de la pauvreté doit aider à rassembler volonté et force de casser les murs». Brigg Morgan dans son travail sur la Grameen Bank montre comment cette discipline financière s'est transformée en un véritable rituel (qui n'est que l'expression d'une forme de discipline sociale) quand, par exemple, chaque semaine l'agent de crédit arrive devant le groupe rassemblé pour lui remettre le remboursement des prêts, comment celui-ci se lève, le salut et récite le slogan de la Grameen : "Discipline, Unity, Courage, and Hard Work " (Morgan, 2006, p. 79).

L'invocation de la discipline ne doit certainement pas laisser indifférents les hiérarques communistes et leur crainte doit assurément être que la microfinance ne crée en Chine des zones de liberté qui les insupporteraient. C'est inversement l'image d'un tel foisonnement d'institutions microfinancières multiples et plurielles, diversement préparées, diversement motivées que renvoie une Inde qui semble alors bien démocratique.

\section{Des mots de la Banque mondiale aux figures de la Banque mondiale : mais où sont les pauvres en Chine?}

Dans un travail récent (Pairault, 2005), nous montrions comment le gouvernement chinois réprimait, récupérait et encadrait les pratiques microfinancières tant traditionnelles que modernes. Il semble qu'une évolution se dessine depuis peu en faveur d'une plus grande acceptation de ce qui pourrait constituer un empiétement oh combien limité ! - sur le pouvoir financier du gouvernement chinois et du parti qui le commande. La Chine manipule donc avec précaution le concept de microfinance et lui préfère souvent celui de finance populaire dont la définition lui appartient et dont le contenu peut varier au gré des politiques (Pairault, 2009, pp. 9-35). Toutefois, dans le dialogue qu'elle entretient avec les agences internationales, elle reste obligée d'utiliser le vocabulaire de celles-ci et prétendre adhérer à leurs objectifs. 


\subsection{Un bilan}

Les IMF chinoises auraient été environ 300 en 2005-2006. Par comparaison, au Bénin qui compte deux cent vingt fois moins d'habitants que la Chine, on recense 762 IMF! L'écrasante majorité des IMF chinoises ( $80 \%$ des projets) semble être gérée dans le cadre de programmes financés par des organisations internationales comme le PNUD, l'UNICEF (Fonds des Nations unies pour l'enfance ou United Nations Children's Emergency Fund), la Banque mondiale, etc. Ces projets manifestent l'ouverture quasi obligée de la Chine à ces institutions. Ils comportent tous un volet " microcrédit " même si ce ne semble pas être le plus souvent leur vocation principale. Le cas emblématique est celui de l'IFAD (International Fund for Agricultural Development) ou FIDA (Fonds international de développement agricole) dont l'aide est considérée comme relative au développement agricole plutôt que relative à la lutte contre la pauvreté par le microcrédit. Par ailleurs, il apparaît distinctement que ces programmes sont très majoritairement de 'vieux' programmes (datant du milieu des années 1990) et donc leurs objectifs microfinanciers sont excessivement limités. Les projets minoritaires seraient eux gérés par des ONG chinoises à travers leur réseau de promotion du microcrédit. Il s'agit de fait d'organisations plus para que non gouvernementales car elles dépendent du ministère du Commerce, de la Fédération panchinoise des femmes, de l'Académie des sciences sociales de Chine ou encore des autorités locales. Il faudrait encore y ajouter d'une part les expériences de microcrédit menées dans le sud-ouest dans le cadre de la lutte pour l'éradication de la pauvreté, d'autre part les activités de quelques ONG internationales. Bref, toutes ces actions et programmes représentent non seulement peu de choses à l'échelle de la Chine, mais encore moins quand elles sont comparées à l'Indonésie un pays de 240 millions d'habitants où on recenserait quelques cinquante mille IMF dont la Bank Rakyat Indonesia aurait environ trois millions et demi de clients (Thangada, 2009). 


\section{Microcrédit en Chine}

1980-1992 : Aides variées sans contrepartie financière

1993 Création de la Coopérative pour l'éradication de la pauvreté par l'Institut de développement rural de l'Académie des sciences sociales de Chine suivant le modèle bangladeshi de la Banque des villages (traduction littérale de Grameen Bank ; en chinois xiangcun yinhang). [Lin Yifu, l'actuel Premier vice-président et Économiste en chef à la Banque mondiale, participa financièrement, avec d'autres chercheurs, à la constitution d'un fond expérimental de lutte contre la pauvreté au Shanxi].

1995 Le PNUD avec le Centre international de Chine pour les échanges économiques et techniques (activité interrompue depuis 2004-2005 ?) entreprennent de lutter contre la pauvreté par le microcrédit en instaurant des IMF émulant le modèle bangladeshi.

1998 Dotations d'organisations diverses de l'ONU : trois milliards de dollars pour lutter contre la pauvreté.

2000 Les coopératives rurales de crédit sont encouragées à pratiquer le microcrédit avec la possibilité de refinancement auprès de la banque centrale. Entre 2000 et 2006, sur 920 milliards de yuan de crédit octroyés, seuls $18,6 \%$ l'ont été sans garantie d'aucune sorte.

2004 Publication d'un premier texte officiel évoquant le microcrédit et encourageant une réflexion sur ce thème.

2005 La banque centrale prône le développement d'IMF commerciales dans le cadre de la lutte contre la pauvreté.

2006 La commission de contrôle des banques émet des recommandations pour le développement de banques rurales pour soutenir la construction socialiste de nouveaux villages.

2007 Lancement de banques de bourg et de village, cunzhen yinhang, dans les districts les plus pauvres dont celui de Yilong.

2008 La Commission chinoise de contrôle des banques et la banque centrale publient des Orientations relatives aux expériences de sociétés de microcrédit.

2009 Liu Kegu, vice-gouverneur de la Banque nationale de développement, rejette l'universalisme fondateur de la microfinance qui défend le droit pour tous d'accéder à des services financiers précisément sans contrainte de structure.

\subsection{Microfinance et microcrédit}

La méfiance du gouvernement chinois à l'égard des IMF prend naissance dans leur logique même, laquelle repose le plus souvent sur la croyance en l'omnipotence de l'intérêt privé pour satisfaire des besoins collectifs. Or les autorités chinoises n'entendent nullement favoriser un tel point de vue dont le risque est bien souvent de conduire à une quasi privatisation des actions publiques; elles préfèrent les organiser dans le cadre des communautés (shequ) urbaines et rurales dont elles promeuvent l'organisation. Dès lors le choix d'un vocabulaire devient éminemment politique et, partant, l'expression occidentale " microfinance " ne reçoit aucune traduction littérale en chinois. Le terme le plus souvent adopté pour désigner les activités des IMF modernes est xiao'e xindai signifiant littéralement 
prêt chirographaire de faible montant, soit donc microcrédit au sens strict ${ }^{6}$ et, donc, excluant a priori tout autre forme de microfinance qui impliquerait ipso facto une participation à un jeu social que ne contrôlerait pas les autorités chinoises.

En 2003, le PNUD et les Presses de l'Académie des sciences sociales ont publié une étude des politiques à adopter pour lutter contre la pauvreté par la microfinance (UNDP, 2003). Cet ouvrage bilingue chinois-anglais est d'emblée équivoque. Dans une langue il parle de microfinance, tandis que dans l'autre de xiao'e xindai, c'està-dire de microcrédit chirographaire. Les ambigüités linguistiques qui sont autant de prises de positions politiques - ne se limitent pas à la désignation d'un concept devenu central dans les politiques de développement. C'est aussi l'objet auquel doit s'appliquer ce concept qui subit les traitrises de la traduction. Ainsi le titre anglais parle de réduction de la pauvreté (Poverty Reduction) ce qui devrait se traduire en chinois par l'expression jianpin (troncation de jianya pinqiong). Or la traduction adoptée signifie " aider les pauvres ", fupin (troncation de fuzhu pinqiong), ce qui sonne davantage comme l'organisation de la charité que comme le lancement d'une vraie politique de développement économique et social permettant de lutter contre la pauvreté et soutenir les "vrais pauvres", zhenzheng de qiongren, comme le proclame la China Foundation for Poverty Alleviation, Zhongguo fupin jijinhui, érigée par le ministère chinois de l'Intérieur avec les donations octroyées par Bayer, JPMorgan, Microsoft, Standard Chartered Bank, Shell et autres (CFPA, 2007, p. 7). N'est-ce pas répondre à Muhammad Yunus que discipline et charité vont ensemble, la seconde peut même être la pré-condition de la première - ce que les Jésuites et autre missionnaires avaient déjà compris quand ils œuvraient en Chine au XIX ${ }^{\mathrm{e}}$ siècle ?

Les décalages annoncés dans le titre de l'ouvrage du PNUD trouvent d'autres avatars, par exemple dans les recommandations politiques qui closent la publication. Ainsi, en anglais, il est recommandé de faciliter aux ONG l'exercice d'activités financières (UNDP, 2003, p. 195) ; en chinois, il est seulement préconisé de faciliter cet exercice par des "institutions financières [pratiquant de] petites [transactions] ", xiao'e jinrong jigou (UNDP, 2003, p. 155). Certes, quand les ONG existent, il s'agit le plus souvent d'ONG ... gouvernementales! Plus important est, en revanche, la référence à des "institutions financières" car l'expression suggère très clairement que le rôle d'éventuelles IMF est d'agir dans le cadre d'une réglementation surannée et égrotante à côté des banques d'État ou comme filiales ou agents de celles-ci. L'histoire des maisons de prêt

6 À Taiwan en revanche on parle clairement de weixing [micro] jinrong [finance]. L'expression fait aussi son chemin en Chine populaire, voir par exemple http://mf.cfpa.org.cn/detail.cfm?classid=14. 
sur gage est là pour nous rappeler les difficultés qu'il y a d'entreprendre des activités financières ou para financières en Chine (Pairault, 2002 et 2003).

Le premier texte réglementant les maisons de prêt sur gage en Chine est émis en août 1993, elles reçoivent alors le statut d'" institutions financières spéciales ", teshu jinrong jigou, sous la tutelle de la banque centrale chinoise. Elles étaient considérées comme des institutions financières non bancaires, fei yinhang jinrong jigou car elles étaient autorisées à prêter sur gage mais non à recevoir des dépôts. De surcroît, en 1996, on leur invente une appellation avec le néologisme diadang hang (i.e. métier du prêt sur gage) - construit sur le modèle de l'expression chinoise désignant les banques à l'" occidentale " (yin hang, métier de l'argent) - devant obligatoirement apparaitre dans le nom de chacune des maisons de prêt sur gage enregistrée. Pour discipliner un secteur trop entreprenant et partant menaçant d'ouvrir une brèche dans un monopole d'État, les maisons de prêt sur gage perdent en 2000 leur qualité d'institutions financières spéciales pour n'être plus que de simples entreprises industrielles et commerciales soumises à la loi sur les sociétés mais autorisées à pratiquer le prêt sur gage. Lorsqu'en mai 2008 est enfin publié un texte réglementant la création (autorisée en fait depuis 2005) à titre expérimental d'IMF non autorisées à recevoir des dépôts, les autorités chinoises se gardent de favoriser les mêmes fourvoiements. D'emblée, on parle de sociétés de prêts (en général et non seulement chirographaires) de faible montant, xiao'e daikuan gongsi; ce sont des sociétés commerciales, shangyesxing, et à ce titre elles sont soumises à la loi sur les sociétés excluant ainsi que ce puisse être des ONG et interdisant de même toute assimilation hâtive à de quelconques institutions financières. Même l'expression en langue anglaise les désignant aux instances internationales respecte l'acceptation politique de cette appellation : Microcredit Companies. En outre, la raison sociale de ces nouvelles entreprises doit clairement faire apparaitre leur statut non financier. En revanche, il n'est pas exclu que ces sociétés de prêts puissent se transformer un jour en banques de bourgs et de villages, cunzhen yinhang, soumises à la loi bancaire et aptes à recevoir des dépôts (CBRC, 2007 et 2008).

L'objectif ultime de cette réforme, qui prend prétexte de la lutte contre la pauvreté dans les zones rurales mais aussi du développement des $\mathrm{PME}$, est d'établir un système financier commercial compétitif. L'encouragement le plus visible est l'abaissement légal de l'investissement initial ainsi que l'appel aux capitaux nationaux et étrangers. L'exclusion des ONG de cette part du marché nouvellement ouvert, manifeste davantage encore la volonté du gouvernement chinois d'exercer un contrôle macroéconomique et social strict - et donc d'imposer une discipline. 
Ces dispositions semblent incontestablement favoriser un accès plus aisé des plus démunis aux sources formelles de financement par leur diversification et adaptation. Ce faisant, le gouvernement chinois exprime toujours aussi fermement sa détermination à écarter toute forme relativement autonome de microfinance qui pourrait générer des forces centrifuges de développement. La conception sous-jacente du développement est claire. Selon une vulgate classique, celui-ci ne peut s'accomplir sans la modernisation du secteur financier qui s'accommoderait mal d'institutions financières ambiguës de surcroît de petite taille car big is beautiful. L'" approfondissement financier " permettrait le développement économique qui à son tour permettrait d'éradiquer la pauvreté. C'est tenir bien peu compte de l'effarant accroissement des inégalités en Chine qui, plus que la pauvreté absolue, nourrit un sentiment insupportable d'indigence et favorise une instabilité sociale bien plus grave que ne le feront jamais des IMF - pour ne pas parler d'un secteur financier informel effervescent.

\subsection{Muhammad Yunus et Lin Yifu}

L'expérience qui résumerait le mieux la politique chinoise est sans doute celle menée dans le district de Yilong dans la province du Sichuan. Ce district revendique plusieurs titres de gloire, le premier étant d'être la patrie du maréchal Zhu De - l'un des hiérarques communistes mort en 1976. Le second est d'être l'un des 592 districts les plus pauvres de Chine et à ce titre désigné pour recevoir en priorité l'aide du gouvernement chinois. Yilong est devenu aujourd'hui une vitrine de l'action du gouvernement. L'aventure aurait commencé au début des années 1990 par des expériences menées en liaison avec l'Académie des sciences sociales de Chine. Puis, en 1995, Yilong bénéficie du partenariat qui s'instaure entre les autorités chinoises et le PNUD pour la mise en place d'un programme expérimental de microcrédit. Les informations disponibles divergent. Selon Sarah Tsien (2001) de PlaNet Finance qui a participé au lancement du programme, cette expérience serait "inherently different from a Grameen replication model". Selon d'autres auteurs, comme Scott Rozelle (Rozelle et al., 2004, p. 211), "The Yilong micro-credit program was set up as a Grameen Bankstyle ". La presse chinoise semble confirmer cette seconde hypothèse car elle souligne à l'envi que Muhammad Yunus, avait fait dès 2003 un don de 50000 dollars au programme de microcrédit de l'Association pour le développement rural de Yilong (CPAD, 2007)7.

${ }^{7}$ Créée en 1996 par le gouvernement du district de Yilong et dirigée par sa Commission du commerce extérieur et de coopération économique avec l'étranger, cette association est une "ONG sans but lucratif " fei yinglixing de minjian zhuzhi); www.help-poverty.org.cn/helpweb2/ngoyj/ngoyj2.htm. 
Le $1^{\text {er }}$ mars 2007 naît à Yilong la banque de bourg et de village Huimin (c'est-à-dire Bonne pour le peuple) ; elle est une création ex nihilo et non la transformation d'une structure antérieure. L'actionnaire majoritaire, dont le siège social est à Jincheng (cheflieu du district de Yilong) est la Banque commerciale de la municipalité de Nanchong (dont dépend le district) ; les cinq autres actionnaires (chacun apportant un dixième du capital, soit 200000 yuan, environ $20000 €$ ) sont des grandes entreprises locales à capitaux publics ou para publics. Leur clientèle sera exclusivement composée de paysans, de petits entrepreneurs individuels et de micro-entreprises.

Il est parfaitement clair que l'idéal de l'IMF proche des exclus de la finance est loin d'être la qualité majeure du projet pour lequel on invitait Muhammad Yunus. Ce type de banque ne mérite certainement pas l'appellation de banque populaire - grassroots bank que la presse chinoise traduit littéralement par caogen yinhang. La proximité avec les clients est clairement sacrifiée au profit d'une centralisation des opérations au chef-lieu du district d'autant que l'absence de tout réseau routier entretenu rend les communications très malaisées pour la clientèle. Également, la nature même des actionnaires retenus - banque (à capitaux publics certes mais en partenariat avec des investisseurs étrangers ${ }^{8}$ ) et grandes entreprises - ne saurait être sans conséquence sur la gestion de cette banque dont l'ambition "constitutionnelle " est d'évoluer vers des pratiques la rapprochant de ses grandes sœurs. Si la banque Huimin a bien vu le jour, ce n'est pas le cas de tous les projets similaires. La Hong Kong Shanghai Banking Corporation (HSBC), la Standard Chartered Bank et la Grameen Foundation avaient envisagé de créer une banque de bourg et de village, étant entendu que la Grameen Foundation serait l'actionnaire de référence et dirigerait le projet. Or le règlement chinois relatif aux banques de bourgs et villages interdit (article 25) à une fondation de détenir plus de $10 \%$ du capital d'une telle banque et impose que l'actionnaire de référence soit un établissement bancaire - ce que n'est pas la fondation - détenant au moins $20 \%$ du capital. L'entente était impossible (CBRC, 2007).

Il est donc évident que le souhait du gouvernement chinois n'est certainement pas de favoriser des institutions " populaires " au service des plus démunis mais bien plutôt d'accomplir par des voies adoucies un approfondissement financier au sens le plus conventionnel de l'expression tout en préservant l'autorité et le pouvoir de contrôle du Parti-État sur les orientations du développement économique. Dès lors les proclamations de succès

8 Banque à capitaux publics créée en 2001 et partenaire depuis 2005 de la DEG (Deutsche Investitions- und Entwicklungs-. gesellschaft mbH). 
devront être interprétées non comme un succès de pratiques microfinancières au service des exclus de la finance la plus formelle, mais comme une consolidation du pouvoir du gouvernement chinois sur la distribution du crédit. Muhammad Yunus et l'expérience de la Grameen Bank servent donc à la Chine de caution scientifique et morale à ses politiques. Ainsi Lin Yifu, le nouvel Économiste en chef de la Banque mondiale ${ }^{9}$, décrit tantôt la Grameen Bank comme une "banque rurale de crédit chirographaire" (AHGZXC, 2007) - en insistant parfois fortement sur son caractère commercial (Sina, 2007 ; Xinhua, 2007) - , tantôt comme une banque commerciale des PME (China Daily, 2007). Quand on sait qu'une PME chinoise peut compter de 8 à 2999 salariés dans le secteur des transports et celui de la construction ou de 8 à 1999 dans celui de l'industrie, les références au prix Nobel de la paix 2006 et à son œuvre semblent relever moins d'une recherche de patronage que d'une instrumentalisation. Arrêtons-nous sur la personnalité de Lin Yifu dont la très instructive nomination à la Banque mondiale aurait été acceptée mais non demandée, ni même expressément souhaitée par le gouvernement chinois - contrairement à ce qui se passe aujourd'hui (fin 2009) pour Zhu Min ${ }^{10}$ dont la Chine a clairement fait savoir qu'elle désirait qu'il soit nommé directeur général adjoint du FMI.

The Economist (2008, p. 59) présente cet économiste très médiatique en ces termes: "He [Lin Yifu] believes governments go wrong when they defy the law of comparative advantage [...] The government's first duty, he argues, is "to remove all possible obstacles for the function of free, open and competitive markets". Spoken like a Chicago man ". Sa contribution a un ouvrage récent synthétise on ne peut mieux ses positions dans un chapitre où il analyse les rapports entre efficacité, égalité et croissance (Lin, 2008). Lin Yifu estime que les politiques de redistribution ne doivent constituer qu'un outil d'appoint pour lutter contre les inégalités ; les expériences latino-américaines en auraient montré les méfaits. En revanche, si l'État s'abstenait de toute intervention intempestive, il maximiserait l'efficacité du système ; la croissance économique serait

9 Lin Yifu (ou encore Justin Lin) est né en 1952 à Taiwan et a émigré en Chine populaire en 1979 dans des conditions rocambolesques. Docteur en économie de l'Université de Chicago, il est professeur et directeur-fondateur du Centre chinois de recherche économique de l'Université de Pékin. Il est aussi membre de la Commission consultative du peuple chinois et viceprésident de sa commission économique.

10 Zhu Min est également né en 1952 mais à Shanghai ; il a obtenu son doctorat à Johns Hopkins ; il est entré à la Banque populaire de Chine en 1996 dont il est, en 2009, l'un des directeurs adjoints. Sa nomination au FMI aurait pour but que la Chine participe directement à la nouvelle mission de surveillance des monnaies. 
alors maximum et durable de telle sorte que la distribution primaire - assurée par le libre jeu du marché - organiserait d'elle-même la lutte contre la pauvreté par une augmentation généralisée des revenus individuels : "It is our task to ensure that in the course of development, the income of the poor grows faster than that of the rich, but it should not be accomplished by redistribution " (Lin, 2008, p. 93). Dans ce contexte, la redistribution "should solve the residual problems left by primary distribution. It is worth emphasising that in certain circumstances, redistribution can be helpful" (Lin, 2008, p. 94). Il y a plus qu'une différence de perception entre cette vision qui conçoit la pauvreté en termes purement économiques (la simple détention d'une richesse matérielle) et celle défendue par des gens qui, comme Jean-Michel Servet, la conçoivent d'abord en termes sociaux - exclusion, marginalisation... - (Servet, 2006, pp. 430-435 inter al.). Pour ces derniers, la lutte contre la pauvreté - comprise d'abord comme une exclusion sociale - détermine la réduction des inégalités (de statut plus que de revenu) qui peut à son tour débloquer la croissance économique et permettre le développement. L'arrivée de Lin Yifu à la Banque mondiale marque donc pour la microfinance la confirmation d'une stratégie d'un néo-libéralisme convenu, telle qu'elle est définie dans le rapport Finance for all ? (Beck et Honohan, 2007), en même temps qu'elle peut laisser craindre une remise en cause de ses aspects les plus sociaux.

Le conformisme linien explique-t-il la hâte de Robert Zoellig, directeur de la Banque mondiale, qui déclarait en février 2008 : " J'ai hâte de travailler avec lui [Lin Yifu] sur la croissance et l'investissement en Afrique, les possibilités d'échanges de savoir SudSud et les outils bancaires pour mieux soutenir les pays touchés par les prix élevés de l'énergie et des produits agricoles " (Le Soir, 30 mai 2008, p. 22) ? Le rapport que Martin Ravallion a remis en janvier 2008 à la Banque mondiale éclaire la mission confiée à son nouvel Économiste en chef venu d'un pays en développement qui cherche à se créer une zone d'influence en Afrique : "A number of policy messages worth thinking about in an African context emerge from the literature on how China was so successful in the fight against poverty. [...] But it must not be forgotten that Africa is 48 countries not one. There is no African central government to transmit policy lessons from one place to another. Here the international community, including China, can play an important role " (Ravailllion, 2008, pp. 22-24). Le premier voyage de Lin Yifu dans ses nouvelles fonctions fut donc pour l'Éthiopie où il s'est rendu en juin 2008 et où, à l'évidence, il délivra le message de sa contribution à l'ouvrage précité (WaltaInfo, 2008).

Donc, la Chine parait adopter le vocabulaire et les politiques prônées par la Banque mondiale pour les instrumentaliser au profit d'une discipline financière renforcée et d'une discipline sociale 
pérennisée. À son tour, la Banque mondiale emprunte une figure chinoise reconnue comme néolibérale et emblématique de succès économiques (ce qui, notons-le en passant, n'est malheureusement le cas ni du Bangladesh, ni de Muhammad Yunus) pour mieux vanter ses propres solutions et œuvrer à un ordre mondialisé qui est tout autant le respect de disciplines financière et sociale avérées.

\section{Détournement, réappropriation, de Gandhi à la Banque mondiale}

Longtemps ignorée au profit de l'expérience menée par le charismatique Muhammad Yunus avec sa Grameen Bank au Bangladesh, la microfinance indienne retient régulièrement désormais l'attention des médias. Contrairement à la Chine, l'Inde semble offrir une grande liberté d'action, les IMF y foisonnent et le nombre de clients augmente continûment ${ }^{11}$. Malgré une régulation bancaire encore peu adaptée aux petites structures, les nombreuses expériences microfinancières du secteur privé ne sont que très rarement bouleversées par les diktats des autorités. Néanmoins, le gouvernement fédéral, appuyé par les gouvernements des États fédérés, a su imposer une méthode et une vision et, partant, développer l'un des plus grands programmes de microfinance au monde.

\subsection{Inde : un bilan}

Avec un nombre de clients potentiels oscillant entre 320 millions (estimation la plus basse du nombre de personnes vivant sous le seuil de pauvreté) et 650 millions (estimation du nombre de personnes dépourvues de compte bancaire), le marché indien est devenu le plus important au monde (Mohan, 2006, p. 1 ; Fouillet, 2009, pp. 122-128). Son taux de pénétration (proportion de familles concernées), compris entre 8 et $15 \%$ selon les sources, situe l'Inde dans la moyenne asiatique mais se caractérise toutefois par une très forte croissance ; pour l'année financière 2007, le nombre de clients augmente environ de $80 \%$ et celui du volume des crédits de $40 \%$

11 L'IMF SKS illustre bien cette dynamique. Tandis qu'en mars 2004, SKS comptait 30000 clients actifs pour 11 agences, ils étaient 600000 pour 275 agences en mars 2007 et près de 5,3 millions pour 1627 agences en septembre 2009. À noter que Vikram Akula, ancien consultant pour le cabinet de conseils McKinsey \& Company et fondateur et directeur général de SKS, fit partie de la liste établie par le magazine américain Time des cent personnes ayant le plus influencé le monde durant l'année 2007. 
(Sa-Dhan, 2007, p. 23 ; M-Cril et MIX, 2007, pp. 7-16 ; MIX, 2006, pp. 4-5).

La microfinance indienne est généralement présentée comme un "modèle" spécifique car, environ les trois quarts de l'offre proviendraient d'un système d'adossement bancaire (bank linkage) impliquant autorités publiques, agences bancaires (publiques mais aussi privées), organisations non gouvernementales et, bien entendu, les self-help-groups (groupes locaux de 10 à 20 membres chargés de gérer collectivement collecte de l'épargne et remboursement des prêts ; par la suite SHG). Alors que ce modèle a été initié par d'autres pays dans les années 1970 et 1980 - en particulier aux Philippines et en Indonésie (Seibel, 2006) - , il faut attendre la fin des années 1980 pour que le gouvernement indien apporte un soutien financier aux organisations développant ce type de lien financier entre groupes informels et secteur bancaire.

Les premiers SHG indiens apparaissent en 1985 avec le support de 1'ONG Myrada sous le nom de self affinity groups ${ }^{12}$. Ils sont formés sur la base de groupes informels communautaires préexistants dans les villages (sanghams). La Banque nationale de développement rural et agricole (NABARD) soutiendra financièrement ces expériences à partir de 1987 . Les succès rencontrés permettent à la banque centrale indienne (Reserve Bank of India, RBI), appuyée par des experts de l'IFAD, de concevoir en 1990 une stratégie alternative de crédit s'appuyant sur le développement de SHG. La RBI et la NABARD imposent alors au gouvernement d'utiliser l'infrastructure bancaire existante pour soutenir les organisations de la société civile, et d'axer la logique de leur action sur la collecte de l'épargne plutôt que sur celle de l'octroi de crédit comme une reproduction du modèle de la Grameen Bank l'aurait voulu'13. D'emblée le contraste avec la Chine est saisissant. Certes, dans les

12 Basée à Bangalore, cette organisation est fondée en 1968 et ne démarre ses activités liées au développement rural qu'à partir de 1978 ; les dix premières années de son existence sont dédiées, avec l'aide du gouvernement indien, à l'insertion de 15000 réfugiés tibétains.

13 L'un des apports, trop peu connu, du financial market paradigm porté par D.W. Adams est d'avoir tenté de réhabiliter la fonction économique et sociale du moneylender qui souffrait d'une image négative dans de nombreuses zones à travers le monde (Von Pischke, Adams et Donald, 1983) et notamment en Inde (Harriss, 1983). Cet ouvrage collectif dirigé va véritablement être l'une des pierres angulaires d'une nouvelle vision de la finance rurale reconnaissant la demande de la population des zones rurales pour des solutions en termes d'épargne ; élément au cœur de l'apparition du mouvement des Self-Help Group. Même si cette idée n'était pas nouvelle, elle connut une diffusion très importante via le programme de finance rurale de l'Université de l'Ohio dont les anciens étudiants sont présents dans tous les organes de décision liés à la finance rurale (USAID, FAO, Banque Mondiale, IFPRI, IFAD, etc.) ainsi que dans les fondations privées. 
deux cas c'est à l'initiative d'instances du gouvernement central qu'une stratégie est mise en place. Mais si la Chine semblerait viser principalement le crédit, l'Inde entendrait privilégier l'épargne ; et si la première entend exercer un contrôle strict en limitant la pratique à des institutions financières agréées, la seconde quant à elle favorise fondamentalement le recours à des instances populaires et privées. Ainsi, l'ancien directeur de la RBI et actuel président de la NABARD indiquait que : "the State Governments, the formal financial system and community based organisations can be considered as the three pillars in achieving societal transformation. These three pillars perform distinct but mutually reinforcing roles that can have tremendous synergies" (Thorat, 2006, p. 1).

Le premier projet lancé par un État fédéré voit le jour au Tamil Nadu. En 1990, le Tamil Nadu Women's Empowerment Project ${ }^{14}$ s'engage avec l'aide de Myrada dans une expérience pilote menée dans le district de Dharmapuri. C'est à partir de cette première expérience que la NABARD conçoit le principe de l'adossement bancaire. Une collaboration de 1989 à 1991, entre la NABARD et la RBI, permet ensuite de concevoir un projet pilote lancé dans l'ensemble de l'État en 1992 ${ }^{15}$. Au terme de l'expérience, en 1996, près de 5000 SHG étaient adossés à 95 agences bancaires (Nanda, 1995). Le succès rencontré permit à la NABARD d'étendre ce programme au niveau national. Un fonds fut créé et un programme ambitieux de renforcement des capacités des ONG lancé dont la mise en œuvre est confiée au Micro Credit Innovations Department créé en 1998 par la NABARD. Le démarrage est lent mais un véritable décollage s'observe à partir de 1999 grâce, notamment, à l'appui de la RBI et de ces agences régionales dans les États du Tamil Nadu, de l'Andhra Pradesh, du Maharashtra et du Karnataka, de l'implication de milliers d'ONG, de banques commerciales publiques et privées ainsi que de celle d'agences multilatérales comme l'IFAD, l'UNESCO ou la Banque Mondiale.

Le cas indien est donc marqué par l'intervention très prononcée des puissances publiques. Il diffère toutefois du cas chinois sur plusieurs points car l'interventionnisme indien masque à la fois la mise en place d'un filet de protection contre les conséquences de la déréglementation bancaire des années 1990, et un processus de dépolitisation des organisations de la société civile indienne.

14 Financé par le FIDA en passant par le Tamil Nadu Women's Development Corporation.

15 À cette occasion, la NABARD (1992) édite un guide qui servira de cadre stratégique pour l'implication des banques dans le financement des SHG; ce guide s'appuie sur les expériences conduites par la NABARD et Myrada depuis 1987. 
Revenons sur l'histoire récente du crédit rural en Inde. L'augmentation des déficits publics et extérieurs au cours des années 1980 ayant fragilisé l'économie indienne, le gouvernement fut alors contraint par le Fonds monétaire international (FMI) et la Banque mondiale d'accepter en 1991 un plan d'ajustement structurel (PAS). Outre la dévaluation de la roupie, la hausse des taux d'intérêt, etc., le pays dut s'engager dans un programme d'assainissement des finances publiques, de réformes structurelles et de lutte contre la "répression financière". La libéralisation consécutive du secteur financier indien va profondément modifier la dynamique engagée depuis l'indépendance du pays en 1947 et entraîner, au cours des années 1990, la contraction du réseau bancaire dans les campagnes. Ainsi, de juin 1987 à mars 1992 le taux de bancarisation de la population augmente de $56 \%$ à $76 \%$. Commence alors une période de dé-bancarisation jusqu'en mars 2001 où le taux tombe à $51 \%$. La bancarisation de la population indienne a donc été réprimée par la déréglementation financière engagée dans les années 1990. Le nombre absolu de comptes bancaires, toutes zones confondues, a baissé de près de 13,5 millions, passant de plus de 65,8 millions en 1992 à 52,3 millions en $2001^{16}$. Cette baisse était supportée pour $84 \%$ par les ruraux et, plus alarmant encore, principalement par ceux des zones les plus sous bancarisées avant 1990. De surcroît, cette dé-bancarisation s'est traduite par un renforcement du secteur financier informel en particulier des prêteurs privés - rendant d'autant plus indispensable la mise en place d'une stratégie nouvelle dont le résultat a été que la part de la population possédant un compte bancaire était de nouveau de 75 \%o au 31 mars 2006 - soit le même niveau qu'en mars 1992 (Fouillet, 2009).

\subsection{Programmes subventionnés et appropriation locale}

Antérieurement au lancement du PAS, le gouvernement fédéral s'était reposé sur plusieurs projets de prêts à taux aménagés faisant appel à l'aide internationale pour désenclaver les zones rurales, dont le plus important d'entre eux l'IRDP ou Integrated Rural Development Program (Drèze et al., 1997). Ces expériences présentaient de nombreuses déficiences : taux de recouvrement des prêts très faible, populations visées mal ou peu atteintes, programmes ne subsistant que grâce aux subventions. Face à ces évaluations de plus en plus défavorables et aux cas manifestes de corruption (Drèze 1990,

16 Ce qui malgré tout représentait $21 \%$ de la population ayant un crédit en mars 1992 . 
p. A99), la Banque mondiale, le principal bailleur, décida alors de stopper le financement du projet. Le gouvernement fédéral et surtout les gouvernements fédérés ne pouvaient admettre une telle décision (Copestake, 1996, p. 51) : "The perpetuation of IRDP can best be explained by the political need to perpetuate a myth about bureaucratic capability to reduce mass poverty. [...] the most important audience of the IRDP myth may be the state itself, rather than the wider voting public. ". Le gouvernement indien, cherchant de nouvelles solutions, constata que l'IFAD conduisait des nouveaux projets de crédit rural, les self affinity groups et Gram Kosh du sud de l'Inde. Il décida de participer avec l'IFAD à des études pilotes puis, très rapidement, de lancer à l'échelle nationale ce qui devint le Self Help Group Bank Linkage Program.

Le gouvernement fédéral n'est pas seul à intervenir et les programmes de microfinance sont aussi l'objet d'appropriations "populistes " comme on l'observe dans le cas de l'Andhra Pradesh (Manor, 2000). Pour les uns, cet État était devenu un État libéral modèle; pour les autres, il se serait transformé en un véritable laboratoire pour des réformes économiques néolibérales prônées par de nouveaux Chicago Boys. Quoi qu'il en soit, la politique de Chandrababu Naidu (Chief Minister de l'Andhra Pradesh de 1994 à 2005) a justifié aux yeux de la Banque mondiale qu'elle subventionne la création d'un immense réseau de SHG sur tout le territoire de l'Andhra Pradesh. Deux programmes principaux ont été créés, le District Poverty Initiatives Project et le Rural Poverty Reduction Program plus connus sous leur nom générique de Velugu (la lumière en langue telougou). Ils s'inscrivent dans une architecture de développement plus large nommée "Vision 2020 ", conduite par les chercheurs de l'Institut Adam Smith, la Banque mondiale et le cabinet de conseil McKinsey \& Co. Le Velugu fut si fortement subventionné qu'il permettait des taux d'intérêt très bas, environ $9 \%$ l'an. Dans le même temps, les IMF privées imposaient des taux beaucoup plus élevés - officiellement autour de $24 \%$ mais bien souvent au-delà de ce plafond compte tenu des "coûts cachés " (Fouillet et Augsburg, 2010, p. 199). Après les élections de 2004, le nouveau premier ministre de l'Andhra Pradesh issu de l'opposition (Congress Party), Rajsekhar Reddy, ne fut pas en reste et lança le programme Pavada Vaddi ( $3 \%$ d'intérêt) destiné à compléter les subventions au Velugu et à réduire de 9 à $3 \%$ le coût des crédits aux SHG. Ainsi, lorsqu'un SHG bénéficiait d'un prêt à un taux de $9 \%$, il lui était restitué $6 \%$ une fois le prêt remboursé. Un an plus tard, le gouvernement de l'Andhra Pradesh voulut aller encore plus loin et appela les SHG à ne plus payer que $3 \%$ directement, l'État acquittant le reste.

On est bien loin ici de la discipline financière et sociale généralement mise en exergue. Une lecture rapide pourrait laisser 
entendre que la microfinance ne serait qu'un outil de diffusion des politiques néolibérales alors que la réalité, plus complexe, n'offre pas l'image d'une opposition irréductible entre une microfinance commerciale avide d'autonomie financière - sinon même de profits - et une microfinance solidaire, mais subventionnée et inefficace - pour tout dire ringarde. Certes la pratique actuellement prônée naît des politiques liées au post-consensus de Washington (Weber, 2002, 2006). Toutefois on assiste, comme ici dans l'Andhra Pradesh, à une réappropriation politique locale de cet outil - ce que la pratique politique chinoise exclut d'emblée. Le programme de SHG piloté par la Banque mondiale et par des groupements privés, a pu être détourné par deux premiers ministres successifs de bords différents qui surent utiliser à leurs fins les subventions que versait sans compter la Banque mondiale ${ }^{17}$.

\subsection{Bhoodan Movement et appropriation zélatrice}

Les instances politiques ne sont pas seules à s'approprier les pratiques microfinancières. Au Tamil Nadu, l'organisation Assefa en offre un exemple criant. Assefa est issue du mouvement Bhoodan créé par une haute figure religieuse indienne Vinoba Bhave (18951982). Alors qu'il étudie le sanskrit à Bénarès, Bhave rejoint Mahatma Gandhi comme disciple et organise la résistance face aux Britanniques; il passera près de cinq années en prison. Après l'assassinat de Gandhi (1948), Bhave, qui apparaît comme son légitime successeur, fonde en 1951 le Bhoodan Movement (ou landgift movement). Les partisans de ce mouvement populaire voyageront des milliers de kilomètres à pied à travers le pays, acceptant des donations de terres afin de les redistribuer aux sans-terre. En 1969, le Bhoodan aurait collecté plus de 1,6 million d'hectares de terres à redistribuer. Sri Loganathan, l'un des compagnons de Bhave, fonde Assefa en 1969 pour organiser le développement rural et la défense des petits paysans dans le Tamil Nadu. Avec Assefa, il tente de promouvoir des communautés d'agriculteurs travaillant une terre collective et combattant toute forme de servitude en milieu rural. Il cherche aussi à encourager la reconstruction sociale et économique prônée par Gandhi et à susciter de nouvelles formes de relations sociales: comme "le progrès de tous" (le sarvodaya) en encourageant des mariages inter-castes et interreligieux, ou comme les Pada Yatra (marches aux pieds nus) visant à construire une

17 C'est près de deux milliards de dollars qui ont été empruntés par le gouvernement de l'Andhra Pradesh auprès du Département for International Development de la Banque mondiale, soit près d'un tiers de l'ensemble des fonds pour l'Inde (Sen et Franket, 2005, p. 21).

134 Economie et Institutions $-\mathrm{n}^{\circ} 14-1^{\mathrm{e}}$ semestre 2010 
conscience politique parmi la communauté, ou encore comme le partage permanent des connaissances et des expériences à travers des séminaires, des conférences, etc. C'est aussi la promotion de nouvelles formes de démocratie locale (à travers les Gram Sabha ou assemblées de village) et de développement local (à travers les Gram Kosh ou caisses locales mutualistes).

Assefa n'a pas sous-estimé le phénomène de la microfinance et a si bien su prendre ce tournant qu'elle a fait partie des premières organisations à lancer des SHG. Il existe des différences essentielles entre les Gram Sabha, Gram Kosh d'une part et les SHG d'autre part. Les premiers, développés sur la base de caisses villageoises ${ }^{18}$, ont toutefois été divisés en SHG tant et si bien qu'il est parfois devenu très difficile d'observer une différence dans leur mode de fonctionnement apparent (y compris dans leur course aux subventions) ${ }^{19}$ et ce d'autant plus qu'Assefa évite le plus possible de manifester tout activisme quand elle participe à des instances nationales et internationales et cale son discours sur celui du courant dominant. En revanche, au niveau local, Assefa continue de manifester un inlassable prosélytisme notamment à travers l'organisation d'assemblées réunissant des dizaines de milliers de personnes autour du concept gandhien de Sarvodaya et, donc, parvient à récupérer au profit de sa mission le développement de la microfinance.

Hormis le cas de cette organisation qui se démarque par son appartenance à un important mouvement social, le développement de la microfinance ces dernières années met souvent à mal le caractère politique et solidaire des ONG indiennes (Kalpana, 2005). Agissant comme mesure de protection contre la déréglementation initiée en Inde par le PAS dans les années 1990, la microfinance serait en partie responsable de la dépolitisation de la solidarité non gouvernementale (Harriss, 2001, Guérin et al., 2007). La croissance de la microfinance s'effectue sur fond de slogans simplistes arguant de ses multiples vertus en matière de création d'auto-emploi, de lutte contre la pauvreté et de lutte contre des inégalités sociales, en particulier de genre. Certaines organisations s'en sont même saisies pour solliciter des subventions. D'autres y ont été fortement incitées par les autorités publiques. D'autres encore, notamment celles qui étaient spécialisées jusque-là sur la défense des droits des minorités, considèrent que la microfinance serait une stratégie des autorités pour les détourner délibérément de leur mission initiale. Même si la

18 Caisse villageoise non obligatoirement monétarisée mais donnant la possibilité de payer sous d'autres formes.

19 Par endroits il est possible d'observer que les communautés originelles n'ont pas disparu et que les SHG n'existent que sur le papier pour obtenir des financements immédiatement réinjectés dans la caisse villageoise. 
principale caractéristique de la microfinance indienne réside dans sa multiplicité et sa pluralité, il est certain que la liste de ses dysfonctionnements est infinie et la dimension populiste et démagogique de certains programmes publics est évidente ${ }^{20}$.

\section{Conclusion}

La microfinance est à la mode. Au premier rang de ses laudateurs figurent les bailleurs de fonds et les organisations internationales qui ont trouvé là un formidable outil pour propager leur version d'un consensus de Washington renouvelé autour de la croyance en l'omnipotence de l'intérêt privé pour satisfaire des besoins collectifs. Ensuite, dépendants des premiers, viennent les praticiens qui, n'ayant pas toujours le recul nécessaire pour juger de leur action, appliquent des formules sans véritablement en percevoir l'enjeu. Il est souvent difficile de percevoir les conséquences sociales de stratégies conçues de bonne foi. L'urgence aggrave encore cette difficulté. En janvier 2005, lorsque les familles de villages entiers furent déplacées suite au raz-de-marée qui frappa les côtes des pays de la baie du Bengale, qui pouvait imaginer que l'allocation de centaines de microcrédits était viciée faute d'avoir tenu compte des structures de pouvoir existantes ? Qui pouvait conjecturer que ces micro-prêts allaient non pas faciliter le remplacement du capital détruit par le raz-de-marée et relancer une activité économique locale mais au contraire détruire davantage encore le tissu communautaire d'entraide et de solidarité ? Ce qui tracassait le plus les familles après la catastrophe, n'était pas d'avoir perdu leur hutte (qu'elles pouvaient assez facilement reconstruire), mais d'avoir perdu les carnets de compte où étaient soigneusement notés toutes les relations de dettes qui les liaient au reste de la communauté. Ainsi, un couple perdait non seulement la liste de tous les présents reçus lors de son mariage, mais aussi le montant des sommes dues pour l'organisation des cérémonies de leurs filles, etc. Ni l'importance des crédits, ni les "bonnes intentions" des uns et des autres, ni la "discipline " des clients dans la gestion de leurs prêts ne suffisent pour combattre la pauvreté. À ce constat, la Chine répond par une attitude dont l'ambigüité peut signifier qu'elle accepte les principes du néo-libéralisme mais en subordonne l'application au respect du centralisme démocratique qui substitue une discipline à une solidarité sociale et politique entre l'État et la société que le Parti

20 Lire notamment cette description de la crise qui a frappé la microfinance dans l'État de l'Andhra Pradesh au début de l'année 2006 (Fouillet et Augsburg, 2009). 
communiste chinois a échoué à réaliser de 1949 à aujourd'hui. En revanche l'Inde, sagement disciplinée, a si bien expérimenté ces principes du néo-libéralisme qu'elle encouragerait aujourd'hui des expériences - peut-être plus démocratiques - qui préserveraient sans doute mieux le tissu communautaire sans pour autant que ce soit au réel détriment de la doxa financière.

Le champ de la microfinance peut se comprendre tant comme celui d'" une multiplicité d'acteurs luttant pour la domination du champ " (Labie, 1998, p. 88) que comme celui d'une unicité d'acteurs protégés dans leur monopole. Et, au-delà d'une norme conçue à Washington, les idiosyncrasies nationales, régionales, locales, etc. arrivent à s'approprier les Best Practices. L'écueil actuel pourrait être non plus celui du consensus de Washington, mais celui du consensus qu'imposeraient les États à leurs circonscriptions territoriales ou encore l'absence de consensus national faute aux États de pouvoir en instaurer un.

\section{Références bibliographiques}

AHGZXC (2007), www.ahgzxc.gov.cn/detail/news.asp?id=1103 [en chinois].

Beck T. et P. Honohan, (2007), Finance for all? Policies and pitfalls in expanding access, Washington DC, World Bank.

CBRC(2007), www.cbrc.gov.cn/chinese/home/jsp/docView.jsp?docID=2 0070129B3A3723 DBDD5B764FF791DE6A05E4D00 [en chinois]. CBRC(2008),www.cbrc.gov.cn/chinese/home/jsp/docView.jsp?doc ID=2008050844C6FDE83536CF44FFF6E85E5BC32C00 [en chinois]. CFPA (2007), Rapport 2007 sur le microcrédit [en chinois], fupin.org.cn/upfiles/200858193 623.pdf CGAP (2002), "Microfinance, grants and non-financial responses to poverty reduction: Where does microcredit fit? ", Focus Note, $\mathrm{N}^{\circ} 20$, Washington D.C., Consultative Group to Assist Poorest. China Daily (2007), www.chinadaily.com.cn/bizchina/200701/08/content_777310_3.htm.

Copestake J., (1996), " The resilience of IRDP: Reform and perpetuation of an Indian myth ", Development Policy Review, XIV (1), pp. 51-68. CPAD (2007), www.cpad.gov.cn/data/2007/0301/article_332947.htm [en chinois].

Daley-Harris S., (2007). État de la campagne du sommet du microcrédit. Rapport 2007, Washington D.C., Campagne du Sommet du Microcrédit. Drèze J., (1990), " Poverty in India and the IRDP delusion ", Economic and Political Weekly, XXV (39), pp. A95-A104. 
Drèze J., P. Lanjouw et N. Sharma, (1997), Credit in Rural India: A Case Study, Discussion Paper $\mathrm{N}^{\circ}$ 6, Development Economics Research Programme, Londres, London School of Economics.

Fernando J., (2006), Microfinance: perils and prospects, New-York et Londres, Routledge.

Fouillet C., (2009), " Microfinance, agriculture et analyse spatiale : le cas de l'Inde ", dans S. Morvant-Roux (éds.), Exclusion et liens financiers, Paris, Economica, pp. 111-132.

Fouillet C. et B. Augsburg, (2010), "La microfinance, un objet de pouvoir de plus ? Conflit en Andhra Pradesh ", dans J. Baixas, L. Dejouhanet et P.-Y. Trouillet (éds.), Conflits et rapports sociaux en Asie du Sud, Paris, L'Harmattan, pp. 189-203.

Guérin I., C. Fouillet et J. Palier, (2007), " La microfinance indienne peut-elle être solidaire ? ", Revue Tiers Monde, CXC, pp. 291-308.

Harriss B., (1983), " Money and commodities: Their interaction in a rural Indian setting ", in J.D. Von Pischke, D.W. Adams et G. Donald (eds.), Rural financial markets in developing countries: Their use and abuse, Baltimore, The Johns Hopkins University Press, pp. 233-241. Harriss J., (2001), dePoliticising Development. The World Bank and social capital, London/New-Delhi, Anthem Pres/LeftWord Books.

Kalmi P., (2007), " The disappearance of cooperatives from economics textbooks ", Cambridge Journal of Economics, XXXI (4), pp. 625-647.

Kalpana K., (2005), "Shifting trajectories in microfinance discourse ", Economic and Political Weekly, XL (51), pp. 17-23.

Labie M., (1998), "La théorie du champ en micro-finance : perspectives d'application ", Notes de recherche, Réseau thématique "

Entrepreneuriat ", Paris, UREF-AUPELF, pp. 66-98.

Lin Y., (2008), "Balancing Equity and Efficiency for Sustained

Growth ", in L. Song et W.-T. Woo (eds.), China's Dilemma: Economic

Growth, The Environment and Climate Change, Canberra: Asia Pacific Press, ANU E Press, Brookings Institution Press, Social Sciences

Academic Press, pp. 90-109.

Manor J., (2000), « Small-Time Political Fixers in India's States : Towel over Armpit' ", Asian Survey, XL (5), pp. 816-835.

M-Cril et MIX (2007), India microfinance review 2007,

Gurgaon/Washington DC, Micro-Credit Ratings International

Limited/Microfinance Information eXchange.

MIX (2006), Benchmarking Asian Microfinance 2006, Washington, DC, Microfinance Information eXchange.

Mohan R., (2006), Economic growth, financial deepening and financial inclusion (Address at the Annual Bankers' Conference, Hyderabad) ; www.bis.org/review/r061121e.pdf.

Morgan B., (2006), " Discipling the developmental subject: neoliberal power and governance through microcredit ", in J. Fernando J. (eds.), Microfinance: perils and prospects, New-York et Londres, Routledge, pp. 64-88. 
Nabard (1992). Guidelines for the pilot project for linking banks with self help groups, Mumbai, National Bank for Agriculture and Rural Development.

Nanda Y.C., (1995), " Country Report India ", APRACA-GTZ Regional Workshop on the Linkage Program, Bangkok, APRACA.

Pairault Th., (2002), "Les habits neufs des maisons de prêt sur gage chinoises ", Mondes en développement, XXX (118), pp. 21-38.

Pairault Th., (2003), "Maisons de prêt sur gage en Chine : une visite de Chongqing ", Anthropologica, XLV (2), pp. 283-291 [19 photos illustrent l'article].

Pairault Th., (2005), " Pratiques microfinancières en Chine : entre répression et récupération ", dans I. Guérin, K. Marius-Gnanou, Th. Pairault et J.-M. Servet (éds.), La microfinance en Asie : entre traditions et innovations, Paris, Karthala, pp. 31-60.

Pairault Th., (2009), Pratiques populaires et microfinancières en Chine, Paris, EAC/AUF.

Ravaillion M., (2008), Are There Lessons for Africa from China's Success against Poverty?, World Bank Policy Research working paper $\mathrm{N}^{\circ} 4463$, Washington D.C., World Bank.

Rozelle S., H. Li et L. Zhang, (2004), " Micro-Credit Programs and OffFarm Migration in China ", Pacific Economic Review, IX (3), pp. 209-223. Sha-Dhan(2007), Quick Report 2007:

A Snapshot of Microfinance Institutions in India, New Delhi, Sa-Dhan. Seibel H. D., (2006), " De la microfinance informelle au linkage banking : interactions entre théorie et pratique ", Dialogue, XXXVI, pp. 49-63.

Sen S. et F. Frankel, (2005), Andhra Pradesh's Long March Towards 2020: Electoral Detours in a Developmentalist State, Philadelphia, University of Pennsylvania. [Center for the Advanced Study of India]. Servet J.-M., (2006), Banquiers aux pieds nus, Paris, Odile Jacob. Sina (2007), finance.sina.com.cn/money/bank/bank_hydt/20070329/ 14533453800.shtml [en chinois].

Thangada G., (2009), Microcapital Story: Key Indonesia Microfinance Player Bank Danamon Raises USD 362m in Capital, www.microcapital.org/microcapital-story-key-indonesia-microfinanceplayer-bank-danaman-raises-usd-362m-in-capital/.

The Economist (2008), Lin's long swim: The World Bank, The Economist, CCCLXXXVI (8563), p. 59.

The Norwegian Nobel Institute, (2006), http://nobelprize.org/nobel_priz es/peace/laureates/ 2006/press.html

Thorat Y.S., (2006), "Financial inclusion and millennium development goals ", Reserve Bank of India Monthly Bulletin, February, www.rbi.org.in/scripts/BS_ViewBulletin.aspx.

TsienS.,(2001), topics.developmentgateway.org/chinamicrofinance/rc/fi ledownload.do?item Id=268552. 
UNDP (1997), " UNDP microfinance assessment report for China ", www.uncdf.org/English

/microfinance/uploads/country_feasibility/chinadb3.pdf.

UNDP (2003), Poverty Reduction, Microfinance, Beijing, CASS, 2003.

Von Pischke, J.D., D.W. Adams et G. Donald, (1983) (eds.), Rural

financial markets in developing countries: Their use and abuse,

Baltimore, The Johns Hopkins University Press.

WaltaInfo (2008), www.waltainfo.com/index.php?option=com_conten

t\&task=view\&id $=656$ \&Itemid $=82$

Weber H., (2002), "The imposition of a global development

architecture: the example of Microcredit ", Review of International Studies, XXVIII (3), pp. 537-555.

Weber H., (2006), " The global political economy of microfinance and poverty reduction: locating local livelihoods in political analysis ", in J. Fernando (eds.), Microfinance: perils and prospects, New-York et Londres, Routledge, pp. 43-63.

World Bank (2002), Donor Brief, 2, www.cgap.org/gm/document1.9.2413/DonorBrief_02.pdf.

Xinhua, (2007), news.xinhuanet.com/video/2007/3/09/content_58 24031.htm [en chinois].

140 Economie et Institutions $-\mathrm{n}^{\circ} 14-1^{\mathrm{e}}$ semestre 2010 